\title{
Acute Hydramnios with Notes of a Case Occurring with Uniovular Twins about the I8th Week of Pregnancy.
}

\author{
By G. Balfolr Marshald, M.D., C.M., F.R.F.P.S.G., \\ Senior Gyncecological Surgeon, Royal Infirmary, Glasgow; \\ Extramural Lecturer in Obstetrics and Gynacology, Glasgow.
}

THERE are two distinct clinical varieties of hydramnios-the acute and the chronic, and to these may be added a third in which the chronic suddenly becomes acute.

Acute hydramnios is rare; it usually appears between the 3rd and 6th month of pregnancy, is sudden in its onset, and the accumulation of amniotic fluid is so rapid that it speedily gives rise to great distress.

The chronic variety, on the other hand, is not uncommon, and is probably familiar to most if not all obstetriciaus, as it occurs with a frequency of from 1 in 100 to 1 in 150 pregnancies. It usually shows itself in the latter half of pregnaney, and is gradual in its onset, though the rate of accumulation of fluid varies, some cases having a moderate, others an enormous distension of the amniotic sac.

Chronic hydramnios may only be considered rare when the excess of liquor amnii is so enormous (as in the recorded quantities of 52 and 56 pints), that it is difficult to believe the uterus could be distended to such an extent without rupturing or at all events miscarrying before so much fluid had collected. The distension is, however, gradual, and the abdominal wall and organs have time to adapt themselves to the ever-increasing pressure. That is so doubt the reason why we occasionally see patients delay seeking relief until they have become bedridden from dyspnoa and interference with the circulation, although the enormous abdominal distension must have been a source of great discomfort.

A rare variety of chronic hydramnios is that form where it aequires an acute character and the distension suddenly begins to increase with great rapidity. I have met with two such cases about the 7th month of pregnancy, the abdomen in both being enormously distended. The history in both was a sudden increase in rapidity of distension with severe abdominal pain confining the patients to bed. As both spontaneously miscarried there was no opportunity of estimating the amount of fluid.

Acute hydramnios is so rare that all cases met with should be 
recorded. Its rarity may be judged by the statistics of Baudelocque's clinic, where the proportion was 8 acute to 623 chronic.

Berkeley and Bonney, in their recent publication, state that they have only seen one example, and that in the 9 th month of pregnancy. This is an unusually advanced month of pregnamcy for acute hydramnios to begin, and one would desire to know the details of the case, as it might be an example of an acute phase occurring during the course of a chronic hydramnios.

Jardine, in his "Clinical Obstetries," gives a good description of one case in which a four months' pregnant uterus became larger than a full-time pregnancy in the space of three weeks.

The seriousness of acute hydramnios is due to its onset in early pregnancy and to the rapid accumulation of amniotic fluid, so rapid indeed that excessive distension occurs in one or two days, and the distension may become so enormous in the course of a few weeks that the patient is cyanosed owing to interference with respiration. The abdominal organs are unable to adapt themselves to the rapid distension, the abdominal wall is stretched to a painful degree, while the patient complains of severe and continuous abdominal pain.

In nearly 50 per cent. of cases of acute hydramnios the pregnancy is one of twins. The twins are usually uniovular, and as a rule only one amniotic sac is affected, the other containing little or even just a trace of liquor amnii.

I have only seen one case of acute hydramnios, which forms the subject of the present paper, and the interest lies in the accuracy of the dates, as I had the patient under observation from the second month of her pregnancy.

Mrs. G., aged 31, and recently married, was first seen by me in consultation on March 7, 1913. Her last menses had occurred from January 1 to 5 , and the question to be decided was one of pregnancy. The patient was of spare build, so there was no difficulty in the diagnosis, the uterus corresponding in size and shape to an eight weeks' pregnancy, which agreed with the period of amenorrhcea. During the examination a small fibroid was felt projecting from the fundus uteri.

On May 9 the patient was again examined as she intended to travel to England the following day, and desired to know if she could do so with safety. The uterus on that date corresponded in size to a normal four months' pregnancy.

On May 11, the day following her arrival in England, she was seized with severe colicy pains in the lower abdomen which confined her to bed. She noticed her abdomen distending rapidly and in two days it was very much swollen. She remained in bed for two wecks, abdominal pain being continuous, and she only rose to return to Glasgow, where I saw her on May 25.

The patient was evidently in great distress owing to the rapid 
distension of the abdomen, and complained of continuous severo dragging pain all over the abdomen and over the sacral region. She also complained of frequent urination though the amount passed each time was small. A catheter specimen of urine had a specific gravity of 1025 , and contained a trace of albumen. The abdomen was much distended by the enlarged uterus which extended to the costal margins, the fundus uteri reaching more than half-way between the umbilicus and sternum. This does not give a true conception of the size of the uterus, which was much larger than an eight months' pregnancy, being round in shape, and both deeper and broader. From symphysis pubis to fundus uteri measured $11 \frac{3}{4}$ in., the body circumference at the level of the umbilicus was $32 \frac{1}{2}$ in. The uterus had the fluctuant character of a large unilocular cystoma, felt very tense and was markedly tender to palpation. It is possible that this tenderness may have been due to stretching of the abdominal wall as the skin was very tense and glistening, but showed neither striæ nor linea nigra. No fotus could be felt either by ordinary palpation or ballottement, nor could a foetal heart be heard. Uterine contractions were distinct, and the uterine soufle well marked.

Vaginal examination showed the usual signs of pregnancy such as port-wine discolouration, softness of vaginal walls and cervix, and marked pulsation in the fornices. The most notable feature ascertained by vaginal examination was the marked bulging of tho lower uterine segment which filled the upper part of the pelvis. The fibroid on the fundus uteri was considerably enlarged and flattened, and distinguished by its consistency.

On May 27 I dilated the cervix uteri to admit the forefinger. The membranes burst before I was prepared and I was only in time to collect a little over five pints of liquor amnii, the rest being lost. At the same time a hypodermic injection of pituitrin was given and the case left to nature. Examination of the uterine cavity with the finger revealed the presence of twins. The uterus contracted well with immediate relief to the patient's previous distress. As the uterine contractions were feeble and the cervix not dilating a second injection of pituitrin was given on the following day-May 28. The action was prompt as the uterus cbntracted well, and first one foetus and then the other were born feet first as far as the neck, but in attempting to extract the necks of both parted leaving the heads in the uterus. They had evidently been dead for some days as they were macerated, this accounting for the easy separation of the trunk from the head although gentle traction was employed. Chloroform was administered and the uterus emptied by the fingers.

Convalescence was uneventful and the uterus involuted woll. On the tenth day the fundus uteri was still palpable through the thin and flabby abdominal wall two inches above the symphysis pubis. The 
fibroid was smaller again, and it was found to be pediculated, mobile and attached just to the left of the middle of the fundus uteri.

Dr. Kennedy examined the placenta and membranes for me, but could not find anything abnormal about them.

The twins were uniovular, both males, and equal in size to a four months old fotus. One amniotic sac only was affected by the hydramnios. Unfortunately the foetuses were destroyed before I had them examined, as it would have been of interest to inspect the heart, kidney and bladder which are stated to be regularly found hypertrophied in the hydramniotic foetus. 


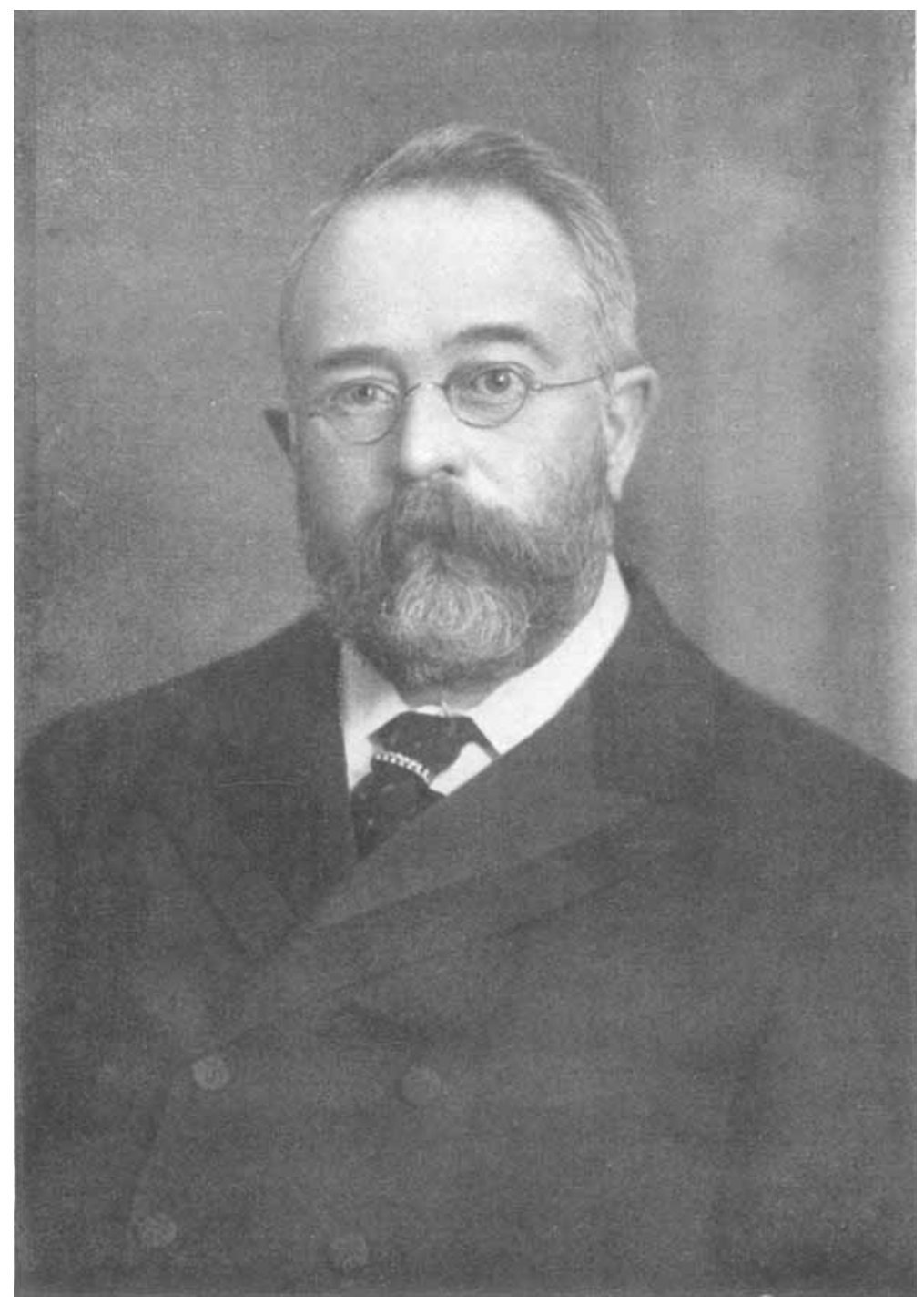

GEORGE ERNEST HERMAN. 